\title{
Markov inequality on sets with polynomial parametrization
}

\author{
by MirosŁaw Baran (Kraków)
}

\begin{abstract}
The main result of this paper is the following: if a compact subset $E$ of $\mathbb{R}^{n}$ is UPC in the direction of a vector $v \in S^{n-1}$ then $E$ has the Markov property in the direction of $v$. We present a method which permits us to generalize as well as to improve an earlier result of Pawłucki and Pleśniak [PP1].
\end{abstract}

1. Introduction. Let $E$ be a compact subset of $\mathbb{R}^{n}$ with nonempty interior. Consider the following two classical problems for polynomials:

- (Bernstein's problem) Estimate the derivatives of polynomials at interior points of $E$;

- (Markov's problem) Estimate the derivatives of polynomials at all points of $E$.

For Markov's problem, the most interesting situation is when $E$ has the Markov property.

A set $E$ is said to have the Markov property if there exist positive constants $M$ and $r$ such that the following Markov inequality holds:

$$
|\operatorname{grad} p(x)| \leq M(\operatorname{deg} p)^{r}\|p\|_{E},
$$

for every $x \in E$ and every polynomial $p: \mathbb{R}^{n} \rightarrow \mathbb{R}$. (Here $\|p\|_{E}$ stands for $\sup |p|(E)$ and $|\cdot|$ denotes the Euclidean norm in $\mathbb{R}^{n}$.)

Markov's inequality plays an important role in the constructive theory of functions. Pawłucki and Pleśniak have shown connections between the Markov property and the construction of a continuous linear extension operator $L: C^{\infty}(E) \rightarrow C^{\infty}\left(\mathbb{R}^{n}\right)$ (see [PP2]). Pleśniak [P] has proved that if $E$ is a $C^{\infty}$ determining compact set in $\mathbb{R}^{n}$ then the existence of such an operator is equivalent to the Markov property. Pawłucki and Pleśniak [PP1]

1991 Mathematics Subject Classification: 32F05, 41A17.

Key words and phrases: extremal function, Markov inequality.

Research partially supported by the KBN Grant 210779101 (Poland) and by the Postdoctoral Grant CRM Bellaterra (Spain). 
showed that the closure of a fat subanalytic subset of $\mathbb{R}^{n}$ has the Markov property. They introduced a class of uniformly polynomially cuspidal subsets of $\mathbb{R}^{n}$ (briefly, UPC) and proved Markov's inequality for them. There are several classes of sets which are UPC. In particular, compact convex subsets of $\mathbb{R}^{n}$ with nonempty interior, fat subanalytic subsets of $\mathbb{R}^{n}$ and sets in Goetgheluck's paper [G] (where a first example of Markov's inequality on sets with cusps was proved) belong to this class.

The UPC sets are compact sets which have a polynomial parametrization satisfying some additional (geometrical) conditions. These conditions imply Markov's inequality.

In this paper we present a new approach to the notion of UPC sets. Observe that

$$
|\operatorname{grad} p(x)|=\sup \left\{\left|D_{v} p(x)\right|: v \in S^{n-1}\right\},
$$

where $S^{n-1}$ is the unit Euclidean sphere in $\mathbb{R}^{n}$, and $D_{v} p$ denotes the derivative of $p$ in the direction of the vector $v$. We shall say that a compact set $E$ has the Markov property in the direction of $v \in S^{n-1}$ if there exist positive constants $M$ and $r$ such that

$$
\left\|D_{v} p(x)\right\|_{E} \leq M k^{r}\|p\|_{E}
$$

for all polynomials of degree $\leq k$. It is clear that having the Markov property is equivalent to the Markov property in $n$ linearly independent directions. It can happen that a set $E$ has the Markov property only in $k, 1 \leq k<n$, linearly independent directions (see Example 4.1). Hence the new notion is indeed more general.

In our investigations a crucial role is played by the following result which is strictly connected with Bernstein's problem.

1.1. Proposition ([B1], [B4], see also [B2]). Let $E$ be a compact subset of $\mathbb{R}^{n}$. Then for all $x \in E$, all $v \in S^{n-1}$ and all polynomials $p$ of degree $\leq k$,

$$
\left|D_{v} p(x)\right| \leq k D_{v+} V_{E}(x) \begin{cases}\left(\|p\|_{E}^{2}-p(x)^{2}\right)^{1 / 2} & \text { if } p \in \mathbb{R}\left[x_{1}, \ldots, x_{n}\right], \\ \|p\|_{E} & \text { if } p \in \mathbb{C}\left[x_{1}, \ldots, x_{n}\right] .\end{cases}
$$

Here $V_{E}$ is the extremal function defined by

$$
V_{E}(z)=\sup \left\{u(z): u \in \mathcal{L}, u_{\mid E} \leq 0\right\} \quad \text { for } z \in \mathbb{C}^{n},
$$

where $\mathcal{L}$ is the Lelong class of all plurisubharmonic functions in $\mathbb{C}^{n}$ with logarithmic growth: $u(z) \leq$ const. $+\log (1+|z|)$ (see $[\mathrm{S}])$, and

$$
D_{v+} V_{E}(x)=\liminf _{\varepsilon \rightarrow 0+} \frac{1}{\varepsilon} V_{E}(x+i \varepsilon v)
$$

(see [B1], [B4]). The above Dini derivatives of the extremal function play an important role in applications to Markov's problem. In the classical situation of $E=[-1,1]$, Proposition 1.1 reduces to the Bernstein (if $p$ is a real 
polynomial) and Markov-Bernstein (if $p$ is a complex polynomial) inequalities.

The paper is organized as follows: in Section 2 we prove the Bernstein and Markov inequalities on a polynomial curve; in Section 3 we define UPC sets in the direction of a vector $v$ and give a Markov type inequality in the direction of $v$-this is the main result of this paper. In the special case of a convex symmetric subset with nonempty interior we obtain another proof of a sharp result which was earlier obtained in [B4]. In Section 4 we give some examples where we apply the results of Sections 2 and 3.

\section{Bernstein and Markov inequalities on a polynomial curve.} Fix $v \in S^{n-1}$. For a given subset $E$ of $\mathbb{R}^{n}$ and $x \in E$, we define the distance of $x$ from $\mathbb{R}^{n} \backslash E$ in the direction of $v$ by

$$
\varrho_{v}(x)=\operatorname{dist}_{v}\left(x, \mathbb{R}^{n} \backslash E\right):=\sup \{t \geq 0:[x-t v, x+t v] \subset E\} .
$$

One can easily verify that if $E$ is compact then $\varrho_{v}$ is upper semicontinuous on $E$. Moreover,

$$
\varrho_{v}(x) \geq \varrho(x):=\operatorname{dist}\left(x, \mathbb{R}^{n} \backslash E\right) \text { and } \varrho(x)=\inf \left\{\varrho_{v}(x): v \in S^{n-1}\right\} .
$$

The following result plays a crucial role in this section.

2.1. Proposition. Let $E$ be a compact subset of $\mathbb{R}^{n}$ and let $\phi: \mathbb{R} \rightarrow \mathbb{R}^{n}$ be a polynomial mapping such that $\phi([0,1]) \subset E$. Put $d=\max (1, \operatorname{deg} \phi)$. Then

$$
D_{v+} V_{E}(\phi(t)) \leq 2 d \sup _{0 \leq r \leq 1} \frac{\sqrt{r(1-r)}}{\varrho_{v}(\phi(r t))}
$$

for $0 \leq t<1$ and $v \in S^{n-1}$.

Proof. Fix $t \in[0,1), \varepsilon>0$ and $R>1$. Assume that the right hand side of the inequality is finite. Denote by $\widetilde{\phi}$ the natural extension of $\phi$ to the whole plane $\mathbb{C}$. Define

$$
f(\zeta)=\widetilde{\phi}\left(\frac{1}{2} a t(g(\zeta)+1)\right)+\frac{i}{2}\left(\zeta-\zeta^{-1}\right) b \varepsilon v
$$

for $|\zeta| \geq 1$, where $g(\zeta)=\frac{1}{2}\left(\zeta+\zeta^{-1}\right)$ is the Joukowski function and $a=$ $2 /(g(R)+1), b=2 /\left(R-R^{-1}\right)$.

Assume for the moment that

$$
f\left(S^{1}\right) \subset E .
$$

Then, by the maximum principle for subharmonic functions and by the definition of $V_{E}$, we obtain $V_{E}(f(\zeta)) \leq d \log |\zeta|$ for $|\zeta| \geq 1$. In particular,

$$
V_{E}(\phi(t)+i \varepsilon v) \leq d \log R .
$$


Now notice that

$$
f\left(e^{i \theta}\right)=\phi\left(\frac{1}{2} a t(\cos \theta+1)\right)-\sin \theta b \varepsilon v
$$

and the condition $f\left(S^{1}\right) \subset E$ is equivalent to

$$
\phi(a t r) \pm 2 \sqrt{r(1-r)} b \varepsilon v \in E \quad \text { for each } 0 \leq r \leq 1 .
$$

This condition will be satisfied if

$$
2 \sqrt{r(1-r)} b \varepsilon \leq \varrho_{v}(\phi(a t r)),
$$

or equivalently,

$$
b \sup _{0 \leq r \leq 1} \frac{2 \sqrt{r(1-r)}}{\varrho_{v}(\phi(a t r))} \leq \frac{1}{\varepsilon}
$$

We have

$$
\begin{aligned}
b \sup _{0 \leq r \leq 1} \frac{2 \sqrt{r(1-r)}}{\varrho_{v}(\phi(a t r))} & \leq \frac{b}{\sqrt{a}} \sup _{0 \leq r \leq 1} \frac{2 \sqrt{a r(1-a r)}}{\varrho_{v}(\phi(a t r))} \\
& \leq \frac{b}{\sqrt{a}} \sup _{0 \leq r \leq 1} \frac{2 \sqrt{r(1-r)}}{\varrho_{v}(\phi(t r))} .
\end{aligned}
$$

Since the right-hand side tends to 0 as $R \rightarrow \infty$, and to $\infty$ as $R \rightarrow 1+$, we may choose $R=R(\varepsilon)>1$ such that

$$
\sup _{0 \leq r \leq 1} \frac{2 \sqrt{r(1-r)}}{\varrho_{v}(\phi(t r))}=\frac{\sqrt{a}}{2 \varepsilon}\left(R-R^{-1}\right) .
$$

It is clear that the condition $f\left(S^{1}\right) \subset E$ is satisfied, and $R \rightarrow 1$ as $\varepsilon \rightarrow 0+$. Now, observe that

$$
\lim _{R \rightarrow 1+} 2\left(R-R^{-1}\right)^{-1} \log R=1 .
$$

By the definition of $D_{v+} V_{E}$ we have

$$
\begin{aligned}
D_{v+} V_{E}(\phi(t)) & \leq d \lim _{\varepsilon \rightarrow 0+} \frac{1}{\varepsilon} \log R(\varepsilon)=d \lim _{\varepsilon \rightarrow 0+} \frac{\sqrt{a}}{2 \varepsilon}\left(R(\varepsilon)-R(\varepsilon)^{-1}\right) \\
& =d \sup _{0 \leq r \leq 1} \frac{2 \sqrt{r(1-r)}}{\varrho_{v}(\phi(r t))} .
\end{aligned}
$$

This completes the proof.

2.2. Corollary. If $x \in \operatorname{int}(E)$, then

$$
D_{v+} V_{E}(x) \leq 1 / \varrho_{v}(x) .
$$

Using a similar argument to that of the proof of Proposition 2.1 one can also prove the following 
2.3. Proposition. Let $\Omega$ be a bounded, star-shaped (with respect to the origin) and symmetric domain in $\mathbb{R}^{n}$ and let $E=\bar{\Omega}$. Then

$$
D_{v+} V_{E}(x) \leq \sup _{0 \leq r \leq 1} \frac{\sqrt{1-r^{2}}}{\varrho_{v}(r x)} \quad \text { for } x \in \operatorname{int}(E),
$$

with equality in the case where $E$ is convex.

Proof. A star-shaped symmetric set has a natural parametrization $t \rightarrow$ $t x, t \in[-1,1], x \in E$. The inequality in Proposition 2.3 is obtained by a similar argument to that of Proposition 2.1 applied to the mapping

$$
f(\zeta)=a g(\zeta) x+\frac{i}{2}\left(\zeta-\zeta^{-1}\right) b \varepsilon v,
$$

where $g(\zeta)$ and $b$ have been defined in the proof of Proposition 2.1 and $a=1 / g(R)$.

Now consider the case where $E$ is convex. Then

$$
E=\left\{x \in \mathbb{R}^{n}: x \cdot w \leq 1, \forall w \in E^{*}\right\},
$$

where $E^{*}$ denotes the polar of $E$. It is easy to see that

$$
\varrho_{v}(r x)=\inf \left\{\frac{1-|r||x \cdot w|}{|v \cdot w|}: w \in E^{*}\right\} .
$$

Hence

$$
\sup _{0 \leq r \leq 1} \frac{\sqrt{1-r^{2}}}{\varrho_{v}(r x)} \leq \sup \left\{\frac{|v \cdot w|}{\left(1-(x \cdot w)^{2}\right)^{1 / 2}}: w \in E^{*}\right\} .
$$

It was proved by the author (see [B1], [B4]) that the right-hand side of this inequality is equal to $D_{v+} V_{E}(x)$. This completes the proof.

We need the following lemma, which is a generalization of the well-known lemma of Pólya and Szegö (see [C]).

2.4. Lemma. Let $p$ be a polynomial in one variable of degree $\leq k-1$. If

$$
|p(t)| \leq\left(1-t^{2}\right)^{-\alpha} \quad \text { for } t \in(-1,1),
$$

where $\alpha \geq 1 / 2$ is fixed, then

$$
\|p\|_{[-1,1]} \leq k^{2 \alpha}
$$

Proof. For $\alpha=1 / 2$ we obtain the Pólya-Szegö lemma. The general case reduces to the case $\alpha=1 / 2$ in the following way. Let $X_{k}=\{p \in \mathbb{C}[t]$ : $\operatorname{deg} p \leq k-1\}$. For $\alpha \geq 0$ we define a norm $\|\cdot\|_{\alpha}$ in $X_{k}$ by

$$
\|p\|_{\alpha}:=\sup \left\{\left(1-t^{2}\right)^{\alpha}|p(t)|: t \in[-1,1]\right\} .
$$

For $\alpha>1 / 2$, we have $\|p\|_{\alpha} \leq\|p\|_{1 / 2} \leq\|p\|_{0}=\|p\|_{[-1,1]}$. Observe that the Pólya-Szegö lemma is equivalent to the inequality $\|p\|_{0} \leq k\|p\|_{1 / 2}$. Since $\left(X_{k},\|\cdot\|_{1 / 2}\right)$ is an interpolation space between $\left(X_{k},\|\cdot\|_{\alpha}\right)$ and $\left(X_{k},\|\cdot\|_{0}\right)$ of 
exact exponent $\theta=1-1 /(2 \alpha)$, i.e. $\|p\|_{1 / 2} \leq\|p\|_{\alpha}^{1-\theta}\|p\|_{0}^{\theta}$, by the Pólya-Szegö lemma we obtain $\|p\|_{0}^{1-\theta} \leq k\|p\|_{\alpha}^{1-\theta}$, which completes the proof.

Now we can formulate the main result of this section.

2.5. Proposition. Let $E$ be a compact subset of $\mathbb{R}^{n}$ and let $\phi: \mathbb{R} \rightarrow$ $\mathbb{R}^{n}$ be a polynomial mapping of degree $d \geq 1$ such that $\phi([0,1]) \subset E$. Fix $v \in S^{n-1}$ and assume that $\operatorname{dist}_{v}\left(\phi(t), \mathbb{R}^{n} \backslash E\right) \geq M(1-t)^{m}$ for $0 \leq t \leq 1$, where $M>0$ and $m \geq 1$ are constants. If $p \in \mathbb{C}\left[x_{1}, \ldots, x_{n}\right]$ and $\operatorname{deg} p \leq k$, then

$$
\left|D_{v} p(\phi(t))\right| \leq \frac{1}{M}(2 d k)^{2 m}\|p\|_{E} \quad \text { for } 0 \leq t \leq 1 .
$$

Pr o of. By Proposition 2.1 we obtain

$$
\begin{aligned}
D_{v+} V_{E}(\phi(t)) & \leq \frac{2 d}{M} \sup _{0 \leq r \leq 1} \sqrt{r(1-r)}(1-r t)^{-m} \\
& \leq \frac{2 d}{M}(1-t)^{-(m-1 / 2)} \quad \text { for } 0 \leq t<1 .
\end{aligned}
$$

It follows from Proposition 1.1 that

$$
\left|D_{v} p\left(\phi\left(t^{2}\right)\right)\right| \leq \frac{2 d k}{M}\left(1-t^{2}\right)^{-(m-1 / 2)}\|p\|_{E}
$$

for $|t|<1$. Since $D_{v} p\left(\phi\left(t^{2}\right)\right)$ is a polynomial of degree $\leq 2 d(k-1)$, combining the last inequality with Lemma 2.4 gives our assertion.

3. Markov inequality on UPC sets. Our considerations suggest a modification of the notion of a UPC set introduced in [PP1].

Let $E$ be a compact subset of $\mathbb{R}^{n}$ and let $m \geq 1$. Given $v \in S^{n-1}$, we shall say that $E$ is $m$-UPC in the direction of $v$ if there exist $E_{0} \subset E$, a positive constant $M$ and a positive integer $d$ such that for each $x \in E_{0}$ one can choose a polynomial map $\phi_{x}: \mathbb{R} \rightarrow \mathbb{R}^{n}$ of degree at most $d$ satisfying

$$
\begin{gathered}
\phi_{x}([0,1]) \subset E \quad \text { and } \quad \phi_{x}(1)=x, \\
\varrho_{v}\left(\phi_{x}(t)\right) \geq M(1-t)^{m} \quad \text { for all } x \in E_{0} \text { and } t \in[0,1], \\
\bigcup_{x \in E_{0}} \phi_{x}([0,1])=E .
\end{gathered}
$$

Applying Propositions 2.1, 2.5 and 1.1 we obtain the following

3.1. TheOrem. Let $E$ be an $m$-UPC subset of $\mathbb{R}^{n}$ in the direction of $v$. Then for every $p \in \mathbb{C}\left[x_{1}, \ldots, x_{n}\right]$ with $\operatorname{deg} p \leq k$ we have

$$
\left\|D_{v} p\right\|_{E} \leq C k^{2 m}\|p\|_{E},
$$

where $C=\frac{1}{M}(2 d)^{2 m}$. 
3.2. Remark. In the special case where $E=\left\{(x, y) \in \mathbb{R}^{2}: 0 \leq x \leq\right.$ $\left.1,0 \leq y \leq x^{p}\right\}$ with $p \geq 1$, Theorem 3.1 was proved by Goetgheluck [G].

3.3. Corollary. Assume that there exist $n$ linearly independent vectors $v_{i} \in S^{n-1}$ such that $E$ is UPC in the direction of each $v_{i}$ (with a constant $\left.m_{i}\right)$. Then there exists a constant $C=C(E)$ such that for each $p \in \mathbb{C}\left[x_{1}, \ldots, x_{n}\right]$ with $\operatorname{deg} p \leq k$ the following Markov inequality holds:

$$
|\operatorname{grad} p(x)| \leq C k^{2 m}\|p\|_{E} \quad \text { for all } x \in E,
$$

where $m=\max _{i=1, \ldots, n} m_{i}$.

3.4. Rem ark. If $E$ is a UPC set in the direction of each $v \in S^{n-1}$ with $E_{0}=E$, with the same family of polynomial mappings $\phi_{x}$ and with the same constants $M$ and $m$, for each $v$, then

$$
\operatorname{dist}\left(\phi_{x}(t), \mathbb{R}^{n} \backslash E\right) \geq M(1-t)^{m} \quad \text { for all } t \in[0,1], x \in E .
$$

This is equivalent to the fact that $E$ is UPC. In this case, by Theorem 3.1 we obtain

3.5. Corollary. If $E$ is an $m-U P C$ subset of $\mathbb{R}^{n}$, then

$$
|\operatorname{grad} p(x)| \leq C k^{2 m}\|p\|_{E}
$$

for all $p \in \mathbb{C}\left[x_{1}, \ldots, x_{n}\right]$ with $\operatorname{deg} p \leq k$, where $C=\frac{\sqrt{2}}{M}(2 d)^{2 m}$.

This corollary improves Pawłucki and Pleśniak's result from [PP1] where the Markov inequality for UPC sets was proved with constant $2 m+2$.

We finish this section by proving a version of the Markov inequality for star-shaped sets.

3.6. THEOREM. Let $\Omega$ be a bounded, star-shaped (with respect to the origin) and symmetric domain in $\mathbb{R}^{n}$ and let $E=\bar{\Omega}$. Assume that

$$
\varrho_{v}(t x) \geq M(1-|t|)^{m} \quad \text { for } t \in[-1,1], x \in \partial E,
$$

where $M>0$ and $m \geq 1$ are constants. If $p \in \mathbb{C}\left[x_{1}, \ldots, x_{n}\right]$ and $\operatorname{deg} p \leq k$, then

$$
\left|D_{v} p(x)\right| \leq \sqrt{2} M^{-1 /(2 m)} k \varrho_{v}(x)^{-(1-1 /(2 m))}\|p\|_{E} \quad \text { for } x \in \operatorname{int}(E)
$$

and

$$
\left\|D_{v} p\right\|_{E} \leq\left(2-\frac{1}{m}\right)^{m-1 / 2} \frac{m^{-1 / 2}}{M} k^{2 m}\|p\|_{E} .
$$

Proof. If $x \in \operatorname{int}(E)$, then $x=t_{0} x_{0}$, where $t_{0} \in[0,1)$ and $x_{0} \in \partial E$. Thus we get $\varrho_{v}(t x) \geq M\left(1-|t| t_{0}\right)^{m} \geq M 2^{-m}\left(\sqrt{1-t^{2}}\right)^{2 m}$, which implies

$$
\sup _{0 \leq r \leq 1} \sqrt{1-t^{2}} \varrho_{v}(r x)^{-1} \leq \sqrt{2} M^{-1 / m} \varrho_{v}(x)^{-(1-1 /(2 m))} .
$$


Applying Propositions 1.1 and 2.3 we obtain the first assertion of the theorem. We also have

$$
\sup _{0 \leq r \leq 1} \sqrt{1-r^{2}}(1-r|t|)^{m} \leq\left(2-\frac{1}{m}\right)^{m-1 / 2} m^{-1 / 2}\left(1-t^{2}\right)^{-(m-1 / 2)}
$$

for $t \in(-1,1)$. Hence we obtain, for all polynomials $p$ with $\operatorname{deg} p \leq k$,

$$
\left|D_{v} p(t x)\right| \leq k \frac{m^{-1 / 2}}{M}\left(2-\frac{1}{m}\right)^{m-1 / 2}\left(1-t^{2}\right)^{-(m-1 / 2)}\|p\|_{E} .
$$

Applying Lemma 2.4 completes the proof.

3.7. Corollary. Let $E=\left\{x \in \mathbb{R}^{n}: f(x) \leq 1\right\}$, where $f$ is a norm in $\mathbb{R}^{n}$. If $v \in S^{n-1}$ and $p$ is a polynomial of degree $\leq k$, then

$$
\left\|D_{v} p\right\|_{E} \leq f(v) k^{2}\|p\|_{E} .
$$

Proof. Let $x \in \partial E, t \in[-1,1]$ and $\tau \in \mathbb{R}$. If $|t|+f(v)|\tau| \leq 1$, i.e.

$$
|\tau| \leq \frac{1-|t|}{f(v)}
$$

then $f(t x+\tau v) \leq 1$. So we have

$$
\varrho_{v}(t x) \geq \frac{1}{f(v)}(1-|t|)
$$

and we can apply Theorem 3.6.

3.8. R e m a rk. It follows from the proof of Theorem 3.6 that the following implication holds: if there exist constants $M>0$ and $m \geq 1$ such that $\varrho_{v}(t x) \geq M(1-|t|)^{m}$ for $t \in[-1,1]$ and $x \in \partial E$, then there exist constants $C>0$ and $1 / 2 \leq \alpha<1$ such that $\sup _{0 \leq r \leq 1} \sqrt{1-t^{2}} \varrho_{v}(r x)^{-1} \leq C \varrho_{v}(x)^{-\alpha}$ for $x \in \operatorname{int}(E)$.

The converse implication is also true.

3.9. Proposition. Let $E$ be a compact, fat $(\overline{\operatorname{int}(E)}=E)$, star-shaped and symmetric (with respect to the origin) subset of $\mathbb{R}^{n}$. Assume that

$$
\sup _{0 \leq r \leq 1} \sqrt{1-r^{2}} \varrho_{v}(r x)^{-1} \leq C \varrho_{v}(x)^{-\alpha} \quad \text { for } x \in \operatorname{int}(E),
$$

where $C>0$ and $1 / 2 \leq \alpha<1$ are constants. Then

$$
\varrho_{v}(t x) \geq C^{-2 m} 2^{-2 m^{2}}(1-|t|)^{m} \quad \text { for } t \in[-1,1], x \in \partial E,
$$

with $m=1 /(2(1-\alpha))$.

Proof. Fix $x \in \operatorname{int}(E)$. By the assumptions,

$$
\varrho_{v}\left(t^{2} x\right) \geq \frac{1}{C} \sqrt{1-t^{2}} \varrho_{v}(t x)^{\alpha} \geq \frac{1}{C} \sqrt{1-t^{2}}\left[\frac{1}{C} \sqrt{1-t^{2}} \varrho_{v}(x)^{\alpha}\right]^{\alpha},
$$


which implies

$$
\varrho_{v}(t x) \geq C^{-(1+\alpha)} 2^{-(1+\alpha) / 2}\left(\sqrt{1-t^{2}}\right)^{1+\alpha} \varrho_{v}(x)^{\alpha^{2}},
$$

and, by recurrence,

$$
\varrho_{v}(t x) \geq 2^{-\left(1+2 \alpha+3 \alpha^{2}+\ldots+k \alpha^{k-1}+k \alpha^{k}\right) / 2}\left(\frac{\sqrt{1-t^{2}}}{C}\right)^{1+\alpha+\ldots+\alpha^{k}} \varrho_{v}(x)^{\alpha^{k+1}} .
$$

Letting $k \rightarrow \infty$ gives

$$
\varrho_{v}(t x) \geq C^{-2 m} 2^{-2 m^{2}}\left(1-t^{2}\right)^{m} \geq C^{-2 m} 2^{-2 m^{2}}(1-|t|)^{m}
$$

for $x \in \operatorname{int}(E)$ and $t \in[-1,1]$. Since $\varrho_{v}$ is upper semicontinuous, this inequality also holds for $x \in \partial E$. The proof is complete.

\section{Examples}

4.1. ExAmple. Let $E=\left\{(x, y) \in \mathbb{R}^{2}:|x|<1,|y| \leq e^{-(1-|x|)^{-1}}\right\} \cup$ $\{(-1,0),(1,0)\}$. If $v=(1,0),(x, y) \in \partial E$ and $\phi(t)=t(x, y)$, then easy calculations show that

$$
1-|t| \geq \varrho_{v}(\phi(t)) \geq \frac{1}{2}(1-|t|) .
$$

By Theorem 3.6 we obtain

$$
\left\|D_{1} p\right\|_{E} \leq 2 k^{2}\|p\|_{E}
$$

where $p$ is a polynomial of degree $\leq k$. However, applying a similar argument to that for Zerner's example [Z] one can prove that Markov's inequality on $E$ does not hold for any positive constant $m$.

4.2. ExAmple. Let $\alpha=\left(\alpha_{1}, \ldots, \alpha_{n}\right)$ where $\alpha_{i} \geq 1, i=1, \ldots, n$. Define

$$
E_{\alpha}=\left\{x \in \mathbb{R}^{n}:\left|x_{1}\right|^{1 / \alpha_{1}}+\ldots+\left|x_{n}\right|^{1 / \alpha_{n}} \leq 1\right\} .
$$

Let $e_{1}, \ldots, e_{n}$ be the standard orthonormal basis in $\mathbb{R}^{n}$. Then

$$
\varrho_{e_{i}}(x)=\left(1-\sum_{j=1, j \neq i}^{n}\left|x_{j}\right|^{1 / \alpha_{j}}\right)^{\alpha_{i}}-\left|x_{i}\right| \text {. }
$$

Let $\beta_{i}=\max _{j \neq i} \alpha_{j}, i=1, \ldots, n$. We have

$$
\begin{aligned}
\varrho_{e_{i}}(t x) & =\left(1-\sum_{j=1, j \neq i}^{n}\left|x_{j}\right|^{1 / \alpha_{j}}|t|^{1 / \alpha_{j}}\right)^{\alpha_{i}}-|t|\left|x_{i}\right| \\
& \geq\left(1-|t|^{1 / \beta_{i}} \sum_{j=1, j \neq i}^{n}\left|x_{j}\right|^{1 / \alpha_{j}}\right)^{\alpha_{i}}-|t|^{1 / \beta_{i}}\left(1-\sum_{j=1, j \neq i}^{n}\left|x_{j}\right|^{1 / \alpha_{j}}\right)^{\alpha_{i}} \\
& \geq\left(1-|t|^{1 / \beta_{i}}\right)^{\alpha_{i}} \geq A_{i}(1-|t|)^{\alpha_{i}},
\end{aligned}
$$


with $A_{i}=\left(\max _{j \neq i} \alpha_{j}\right)^{-\alpha_{i}}, i=1, \ldots, n$, for $t \in[-1,1]$ and $x \in E_{\alpha}$. By Theorem 3.6 we obtain

$$
\left\|D_{i} p\right\|_{E_{\alpha}} \leq\left(2-\frac{1}{\alpha_{i}}\right)^{\alpha_{i}-1 / 2} \alpha_{i}^{-1 / 2}\left(\max _{j \neq i} \alpha_{j}\right)^{\alpha_{i}} k^{2 \alpha_{i}}\|p\|_{E_{\alpha}}, \quad i=1, \ldots, n,
$$

for all polynomials $p$ of degree $\leq k$.

This inequality is sharp in the case where $\alpha_{1}=\ldots=\alpha_{n}=1$ and generalizes the classical Markov inequality (see [B4]).

An easy calculation shows that we also have

$$
\sup _{0 \leq r \leq 1} \sqrt{1-r^{2}} \varrho_{e_{i}}(r x)^{-1} \leq \max \left(1,\left(\frac{\beta_{i}}{\alpha_{i}}\right)^{1 / 2}\right) \varrho_{e_{i}}(x)^{-\left(1-1 /\left(2 \alpha_{i}\right)\right)}
$$

for $x \in \operatorname{int}\left(E_{\alpha}\right), i=1, \ldots, n$. Thus, we obtain the following BernsteinMarkov inequality:

$$
\left|D_{i} p(x)\right| \leq \max \left(1,\left(\frac{1}{\alpha_{i}} \max _{j \neq i} \alpha_{j}\right)^{1 / 2}\right) k \varrho_{e_{i}}(x)^{-\left(1-1 /\left(2 \alpha_{i}\right)\right)}\|p\|_{E_{\alpha}}
$$

for $i=1, \ldots, n, x \in \operatorname{int}\left(E_{\alpha}\right)$, and $p \in \mathbb{C}\left[x_{1}, \ldots, x_{n}\right]$ with $\operatorname{deg} p \leq k$.

4.3. ExAmple. Let

$$
E=\left\{(x, y) \in \mathbb{R}^{2}:|x| \leq 1,|y| \leq(1-|x|)\left[1+\log \frac{1}{1-|x|}\right]^{-1}\right\} .
$$

Let $e_{1}=(1,0), e_{2}=(0,1)$. One can check the following estimates:

$$
\varrho_{e_{1}}(t(x, y)) \geq \frac{1}{2}(1-|t|)
$$

and

$$
\varrho_{e_{2}}(t(x, y)) \geq(1-|t|)\left[1+\log \frac{1}{1-|t|}\right]^{-1},
$$

for $t \in[-1,1]$ and $(x, y) \in \partial E$. The first inequality implies

$$
\left\|D_{1} p\right\|_{E} \leq 2 k^{2}\|p\|_{E}
$$

for any polynomial $p$ of degree $\leq k$. By the second inequality, we obtain

$$
\begin{aligned}
D_{e_{2}+} V_{E}(t(x, y)) & \leq \sup _{0 \leq r \leq 1} \sqrt{1-r^{2}}(1-r|t|)^{-1}\left[1+\log \frac{1}{1-r|t|}\right] \\
& \leq\left(1-t^{2}\right)^{-1 / 2}\left[1+\log 2+\log \frac{1}{1-t^{2}}\right] \\
& \leq\left(1-t^{2}\right)^{-1 / 2}\left[1+\sqrt{5}+\log \frac{1}{1-t^{2}}\right] \\
& \leq\left(1-t^{2}\right)^{-1 / 2}(1+\sqrt{5})\left(1-t^{2}\right)^{-1 /(1+\sqrt{5})},
\end{aligned}
$$


for $t \in(-1,1)$ and $(x, y) \in \partial E$. We now have, for every polynomial $p$ with $\operatorname{deg} p \leq k$,

$$
\left|D_{2} p(t(x, y))\right| \leq(1+\sqrt{5}) k^{2+2 /(1+\sqrt{5})}\|p\|_{E}
$$

for $t \in(-1,1)$ and $(x, y) \in \partial E$, and

$$
\begin{aligned}
& \left|D_{2} p(t(x, y))\right| \\
& \leq \leq\left(1-t^{2}\right)^{-1 / 2} \\
& \quad \times \min \left(1+\sqrt{5}+\log \frac{1}{1-t^{2}},(1+\sqrt{5}) k^{1+2 /(1+\sqrt{5})}\left(1-t^{2}\right)^{1 / 2}\right)\|p\|_{E} \\
& \quad \leq k\left(1-t^{2}\right)^{-1 / 2}(1+\sqrt{5})(1+\log k)\|p\|_{E} .
\end{aligned}
$$

Thus, we obtain $\left\|D_{2} p\right\|_{E} \leq(1+\sqrt{5}) k^{2}(1+\log k)\|p\|_{E}$.

\section{References}

[B1] M. Baran, Bernstein type theorems for compact sets in $\mathbb{R}^{n}$, J. Approx. Theory 69 (1992), 156-166.

[B2] - Complex equilibrium measure and Bernstein type theorems for compact sets in $\mathbb{R}^{n}$, Proc. Amer. Math. Soc., to appear.

[B3] - Plurisubharmonic extremal function and complex foliation for a complement of a convex subset of $\mathbb{R}^{n}$, Michigan Math. J. 39 (1992), 395-404.

[B4] -, Bernstein type theorems for compact sets in $\mathbb{R}^{n}$ revisited, J. Approx. Theory, to appear.

[C] E. W. Cheney, Introduction to Approximation Theory, New York, 1966.

[G] P. Goetgheluck, Inégalité de Markov dans les ensembles effilés, J. Approx. Theory 30 (1980), 149-154.

[PP1] W. Pawłucki and W. Pleśniak, Markov's inequality and $C^{\infty}$ functions with polynomial cusps, Math. Ann. 275 (1986), 467-480.

[PP2] -, - Extension of $C^{\infty}$ functions from sets with polynomial cusps, Studia Math. 88 (1989), 279-287.

[P] W. Pleśniak, Markov's inequality and the existence of an extension operator for $C^{\infty}$ functions, J. Approx. Theory 61 (1990), 106-117.

[S] J. Siciak, Extremal plurisubharmonic functions in $\mathbb{C}^{n}$, Ann. Polon. Math. 39 (1981), 175-211.

[Z] M. Zerner, Développement en série de polynômes orthonormaux des fonctions indéfiniment différentiables, C. R. Acad. Sci. Paris 268 (1969), 218-220.

INSTITUTE OF MATHEMATICS

UNIVERSITY OF MINING AND METALLURGY

AL. MICKIEWICZA 30

30-059 KRAKÓW, POLAND
Current address: INSTITUTE OF MATHEMATICS JAGIELLONIAN UNIVERSITY REYMONTA 4 30-059 KRAKÓW, POLAND E: mail: BARAN@IM.UJ.EDU.PL 